\title{
Multiscale Hydrodynamics in Line Contacts with Different Fluid-Contact Interactions
}

\author{
J. Li ${ }^{1,2}$ and Y. Zhang ${ }^{1 \dagger}$ \\ ${ }^{1}$ College of Mechanical Engineering, Changzhou University, Changzhou, Jiangsu Province, China \\ ${ }^{2}$ Changzhou High Technology Research Key Laboratory of Mould Advanced Manufacturing, Changzhou, \\ Jiangsu Province, China \\ $\dagger$ Corresponding Author Email: engmech1@sina.com
}

(Received January 4, 2021; accepted June 2, 2021)

\begin{abstract}
Multiscale hydrodynamics in line contacts were analyzed from the newly developed multiscale flow equations by respectively considering the weak, medium and strong fluid-contact interactions. In the studied line contact, the surface separation is on the same scale with the thickness of the adsorbed layer on the contact surface, and between the coupled adsorbed layers occurs the continuum fluid flow. The present study shows that when using the developed multiscale flow equations to calculate the surface separation for the weak or medium fluidcontact interactions, the value of the parameter $\mathrm{k}$, which is in the important formulation, should be taken as about 5.0, different from its value 1.0 for the strong fluid-contact interaction. The study also shows that owing to the adsorbed layer effect, for a given operating condition, stronger the fluid-contact interaction, greater the surface separation. The results show the significant effect of the fluid-contact interaction in hydrodynamic line contacts in a quite wide range of the surface separation because of the multiscale hydrodynamic effect.
\end{abstract}

Keywords: Adsorbed layer; Contact; Fluid; Hydrodynamics; Multiscale.

\section{NOMENCLATURE}

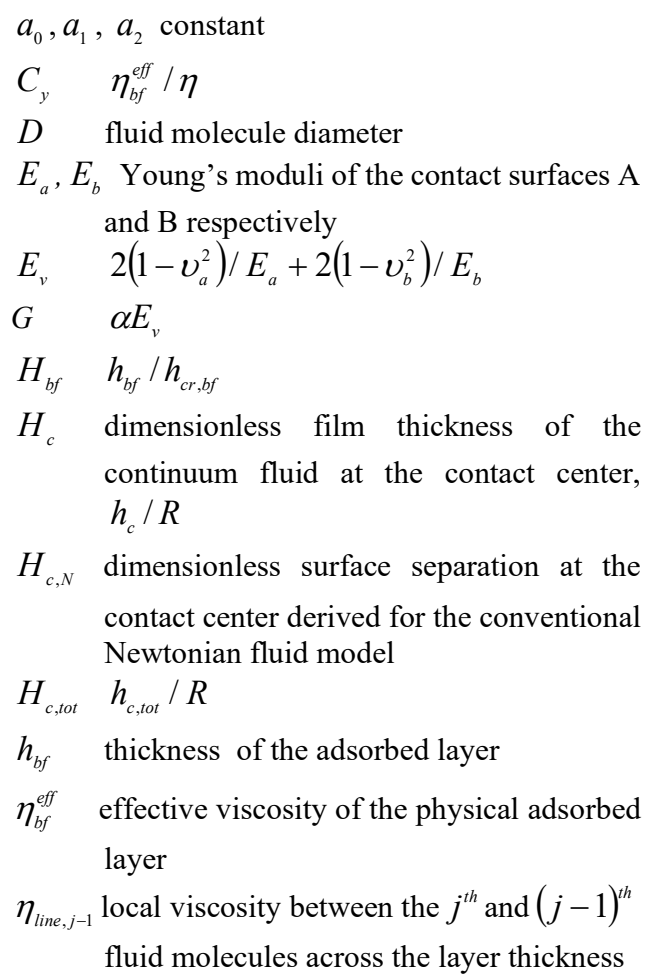
$h_{c}$ dimensional film thickness of the continuum fluid at the contact center




\author{
$\lambda_{b f, e}$ equivalent constant value \\ $v_{a}, v_{b} \quad$ Poisson's ratios of the contact surfaces \\ $\mathrm{A}$ and $\mathrm{B}$ respectively
}

\section{INTRODUCTION}

When the height of a nanochannel is small enough, the nanochannel flow is solely the flow of the adsorbed layer on the channel wall, which is described by the non-continuum nanoscale flow equation (Zhang 2016). The non-continuum, dynamic and interfacial slippage effects of the confined layer should be considered in this flow (Bitsanis et al. 1987; Jabbarzadeh et al.1997; Meyer et al. 1998; Ritos et al. 2014; Sofos et al. 2013; Zhang 2005). In a hydrodynamic line contact such as occurring on gears and roller bearings, such a flow occurring in the extremely small surface separation actually seldom solely occurs because of the existence of the surface roughness. However, it can occur in a local asperity contact in the mixed hydrodynamic line contact where continuum hydrodynamics and physical adsorbed layer noncontinuum hydrodynamics simultaneously occur in different areas of the contact (Begelinger and de Gee 1976; Zhang 2005). In fact, in a local asperity contact, there will also occur the multiscale hydrodynamics where the non-continuum nanoscale adsorbed layer flow is present and between the coupled adsorbed layers occurs the continuum fluid flow. This multiscale hydrodynamics in a local asperity contact is a new hydrodynamic state. For a two-dimensional multiscale flow in a micro/nano slit pore, the flow equations have been derived respectively for the adsorbed layer flow and for the intermediate continuum fluid flow (Zhang 2020). The analysis and calculation have been made for the multiscale hydrodynamics in line contacts considering the strong fluid-contact interaction (Zhang 2021). It was found that for the strong fluidcontact interaction, the value of the parameter $k$, which is in the important formulation $\lambda_{b f, e}=h_{b f} /\left(k h_{c}\right)$, can be taken as 1.0 (Zhang 2021). Very significant deviations from conventional line contact hydrodynamic theories were also observed showing the strong effect of the adsorbed layer in increasing the surface separation (Zhang 2021).

The present study made the calculations for the multiscale hydrodynamics in line contacts when the fluid-contact interaction is respectively mediumlevel and weak, by using the previously developed multiscale analysis (Zhang 2021). Important results have been obtained regarding how to determine the value of the parameter $k$ for these calculations. The results also show how the fluid-contact interaction influences the surface separation for a given load. The study is of significant interest to the research and
$\Delta_{n-2} \quad$ separation between the neighboring fluid molecules across the layer thickness just on the adsorbed layer-fluid interface

$\Delta_{j-1} \quad$ separation between the $j^{t h}$ and $(j-1)^{t h}$

fluid molecules across the layer thickness

design of the mechanical elements such as gears and bearings which are lubricated by very thin oil layers in the condition of heavy loads.

\section{MULTISCALE HYDRODYNAMICS IN A LINE CONTACT}

Figure 1 shows the multiscale hydrodynamic line contact which is elastically deformed under a load; There is an elastically flattened Hertzian contact zone. When the load is heavy enough or the rolling speed is low enough, the surface separation $h_{c, t o t}$ will be on the same scale with the thickness $h_{b f}$ of the adsorbed layer on the contact surface, and the adsorbed layer effect should be significant. In this multiscale hydrodynamics, the adsorbed layer flow is molecular-scale and non-continuum, and between the two adsorbed layers occurs the continuum hydrodynamic flow (with the thickness $h_{c}$ in the Hertzian contact zone). Conventionally, the fluid flow in a hydrodynamic line contact is only considered as the continuum fluid flow by ignoring the existence of the adsorbed layer. While, in the present study, the very thin adhering boundary layers near the contact surfaces are considered as in the non-continuum flow, whereas the continuum fluid flow occurs between the adhering layers of the two contact surfaces. These special flow regimes exist when the surface separation is sufficiently small, but were ignored in the past.

The formation of the adhering layer on the contact surface strongly depends on the interaction between the fluid molecules and the molecules of the contact surface, which may be strong, mediumlevel or weak. The interaction force between the fluid molecules and the molecules of the contact surface can be the van der Waals force or the Coulomb force, the former for non-charged atoms and the latter for charged atoms. For the van der Waals intermolecular force, the interaction strength is determined by the interaction potential, which can result in the weak, medium or strong fluid-contact interactions. For example, for oilphobic contact surfaces such as covered with the coating PTFE (Poly tetra fluoro ethylene), the interaction between the oil and the contact surface is weak, and there may be only a negligible or even vanishing layer on the contact surface. For oilphilic contact surfaces such as covered with specific coatings, the interaction between the oil and the contact surface is medium-level or strong depending on the oilphilicity, and there may be the 


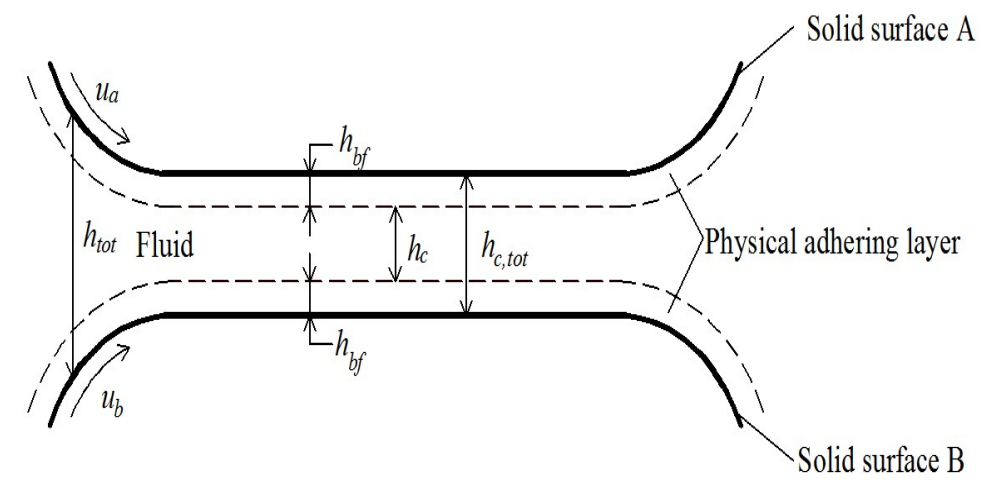

Fig. 1. The studied multiscale hydrodynamic line contact (Zhang 2021).

adhering layer on the contact surface which is much more viscous and dense with several nanometers thickness than for oil-phobic contact surfaces. Also, the molecules within the adhering layer are much more ordered normal to the contact surface for the medium and strong fluid-contact interactions than for the weak fluid-contact interaction, and this results in the much more significant non-continuum effect of the adhering layer for the medium and strong interactions owing to the significantly increased discontinuity and inhomogeneity across the layer thickness.

\section{ANALYSIS}

The adsorption of the fluid molecule layers to the contact surface depends on the fluid-contact interaction. Both the average density and effective viscosity of the adsorbed layer may be increased compared to the fluid bulk values (Abraham 1978; Bocquet and Charlaix 2010; Chauveteau et al. 1984; Horn et al. 1989; Jabbarzadeh et al. 1997; Sofos et al. 2009, 2010). The layer ordering normal to the contact surface leads to the discontinuity and inhomogeneity effects i.e. the non-continuum effect across the adsorbed layer thickness (Zhang 2006). For a weak fluid-contact interaction, the layer may slip on the contact surface (Calabrò et al. 2013; Mattia and Calabro 2012; Sbragaglia et al. 2006).

In the present study, the adsorbed layer flow is described by the flow factor approach model (Zhang 2006), which well incorporates the above mentioned nanoscale flow factors. The flow of the intermediate continuum fluid is described by the Newtonian fluid model. The interfacial slippage is assumed as absent on any interface. Also, the adsorbed layers on the two contact surfaces are assumed as identical. Detailed analytical derivations for the present multiscale hydrodynamics have been shown by Zhang (2021). Here are only repeated the necessary contents.

\subsection{Governing equations for the present multiscale hydrodynamics}

The load-film thickness relation equation in the dimensionless form for the multiscale hydrodynamic contact in Fig. 1 is (Zhang 2021):

$$
\begin{aligned}
& -0.2041\left(\lg \frac{W}{H_{c}}\right)^{2}+0.8876 \lg \frac{W}{H_{c}}+\lg \left[\frac{4 G U}{\pi F\left(\lambda_{b f, e}\right)}\left(\frac{8}{\pi}\right)^{1 / 2}\right]- \\
& \frac{3}{2} \lg W-1.2725=0, \text { for } 0.01 \leq \frac{W}{H_{c}} \leq 200
\end{aligned}
$$

where $\lg =\log _{10}, W$ is the dimensionless load, $W=w /\left(E_{v} R\right), \quad H_{c}$ is the dimensionless film thickness of the continuum fluid in the location where the pressure gradient is vanishing, $H_{c}=h_{c} / R, U$ is the dimensionless rolling speed, $U=\left(u_{a}+u_{b}\right) \eta_{a} /\left(2 E_{v} R\right), G=\alpha E_{v}$, and

$$
\begin{aligned}
& F\left(\lambda_{b f, e}\right)=\frac{1}{12}- \\
& \frac{1}{C_{y}}\left[\frac{F_{2} \lambda_{b f, e}^{2}}{6}-\frac{\lambda_{b f, e}}{1+\frac{\Delta x}{D}}\left(\frac{1}{2}+\lambda_{b f, e}-\frac{q_{0}-q_{0}^{n}}{q_{0}^{n-1}-q_{0}^{n}} \frac{\Delta_{n-2} \lambda_{b f, e}}{h_{b f}}\right)\right] \\
& \frac{F_{1} \lambda_{b f, e}^{3}}{6 C_{y}}+\frac{\varepsilon \lambda_{b f, e}^{3}}{C_{y}\left(1+\frac{\Delta x}{D}\right)}\left(1+\frac{1}{2 \lambda_{b f, e}}-\frac{q_{0}-q_{0}^{n}}{q_{0}^{n-1}-q_{0}^{n}} \frac{\Delta_{n-2}}{h_{b f}}\right)
\end{aligned}
$$

Here, $w$ is the load per unit contact length carried by the contact, $R=R_{a} R_{b} /\left(R_{a}+R_{b}\right), R_{a}$ and $R_{b}$ are respectively the curvature radii of the two contact surfaces, 2/ $E_{v}=\left(1-v_{a}^{2}\right) / E_{a}+\left(1-v_{b}^{2}\right) / E_{b}, E_{a}$ and $E_{b}$ are respectively the Young's moduli of the two contact surfaces, $v_{a}$ and $v_{b}$ are respective-ly the Poisson's ratios of the two contact surfaces, $h_{c}$ is the dimensional film thickness of the conti-nuum fluid in the location where the pressure grad-ient is vanishing, $u_{a}$ and $u_{b}$ are respectively the circumferential speeds of the two contact surfaces, $\eta_{a}$ is the fluid bulk viscosity in the ambient condition, $\alpha$ is the fluid viscosity-pressure index, $C_{y}=\eta_{b f}^{e f f} / \eta, \eta$ is the fluid bulk viscosity, $\eta_{b f}^{e f f}$ is the effective viscosity of the physical adsorbed layer and $\eta_{b f}^{e f f}=D h_{b f} /\left[(n-1)\left(D+\Delta_{x}\right)\left(\Delta_{l} / \eta_{\text {line, }, l}\right)_{a v r, n-1}\right]$, $D$ is the fluid molecule diameter, $n$ is the equivalent number of the fluid molecules across the layer thickness, $\Delta x$ is the separation between the 
neigh-boring fluid molecules in the flow (i.e. horizontal) direction in the adsorbed layer, $(n-1)\left(\Delta_{l} / \eta_{\text {line }, l}\right)_{\text {avr }, n-1}=\sum_{j=1}^{n-1} \Delta_{j-1} / \eta_{\text {line }, j-1}, \Delta_{j-1}$ and $\eta_{\text {line }, j-1}$ are respectively the separation and the local viscosity between the $j^{\text {th }}$ and $(j-1)^{\text {th }}$ fluid molecules across the layer thickness, $q_{0}=\Delta_{j+1} / \Delta_{j}(>1)$, $\Delta_{n-2}$ is the separation between the neighboring fluid molecules across the layer thickness just on the adsorbed layer-fluid interface, $\lambda_{b f, e}$ is an equivalent constant value and

$$
\begin{aligned}
& \lambda_{b f, e}=h_{b f} /\left(k h_{c}\right)(1 \leq k \leq+\infty), \\
& \varepsilon=(2 D I+I I) /\left[h_{b f}(n-1)\left(\Delta_{l} / \eta_{\text {line }, l}\right)_{a v r, n-1}\right], \\
& F_{1}=\eta_{b f}^{e f f}\left(12 D^{2} \psi+6 D \varphi\right) / h_{b f}^{3}, I=\sum_{i=1}^{n-1} i\left(\Delta_{l} / \eta_{\text {line }, l}\right)_{\text {avr }, i}, \\
& F_{2}=6 \eta_{b f}^{e f f} D(n-1)\left(l \Delta_{l-1} / \eta_{\text {line }, l-1}\right)_{\text {avr }, n-1} / h_{b f}^{2}, \\
& I I=\sum_{i=0}^{n-2}\left[i\left(\Delta_{l} / \eta_{\text {line }, l}\right)_{\text {avr }, i}+(i+1)\left(\Delta_{l} / \eta_{\text {line }, l}\right)_{\text {avr }, i+1}\right] \Delta_{i}, \\
& \varphi=\sum_{i=0}^{n-2}\left[i\left(l \Delta_{l-1} / \eta_{\text {line }, l-1}\right)_{a v r, i}+\right. \\
& i\left(\Delta_{l} / \eta_{\text {line }, l}\right)_{\text {avr }, i}=\sum_{j=1}^{i} \Delta_{j-1} / \eta_{\text {line }, j-1}, \\
& \psi=\sum_{i=1}^{n-1} i\left(l \Delta_{l-1} / \eta_{\text {line }, l-1}\right)_{\text {avr }, i}
\end{aligned}
$$

and

$$
i\left(l \Delta_{l-1} / \eta_{\text {line }, l-1}\right)_{a v r, i}=\sum_{j=1}^{i} j \Delta_{j-1} / \eta_{\text {line }, j-1} .
$$

Equation (1) was regressed out from the various numerical calculation results (Zhang 2021). Its accuracy has been verified by comparison with the numerical calculations. It can give a fast solution to $H_{c}$ and is an important tool for the designer of mechanical engineering. The nanoscale behavior of the adsorbed layer flow and the coupling between the non-continuum effect of the adsorbed layer and the continuum hydrodynamic effect are reflected by Eq.(2). Detailed information about Eq.(2) can be found in the reference by Zhang (2021). The multiscale effect is just reflected by the term $F\left(\lambda_{b f, e}\right)$.

For a given operating condition, when $H_{c}$ is solved from Eq.(1), the dimensionless surface separation at the Hertzian contact center is:

$$
H_{c, t o t}=H_{c}+2 \frac{h_{b f}}{R}
$$

Where $H_{c, t o t}=h_{c, t o t} / R$ and $h_{c, t o t}$ is the dimensional surface separation at the Hertzian contact center.

\subsection{For the conventional hydrodynamic line contact}

For comparison, the results from the conventional hydrodynamic line contact theory, which ignores the adsorbed layer and assumes the continuum fluid as Newtonian, are presented in this section. According to the conventional hydrodynamic theory, the dimensionless surface separation $H_{c, N}$ at the Hertzian contact center in the line contact is solved from the following equation (Zhang 2013):

$$
\begin{aligned}
& -0.2041\left(\lg \frac{W}{H_{c, N}}\right)^{2}+0.8876 \lg \frac{W}{H_{c, N}}+ \\
& \lg (24.3825 G U)-\frac{3}{2} \lg W-1.2725=0, \\
& \text { for } 0.01 \leq \frac{W}{H_{c, N}} \leq 200
\end{aligned}
$$

Equation (4) was also regressed out from the numerical calculation results, and it is useful in the design of the corresponding mechanical elements such as gears and bearings lubricated by the Newtonian fluids. Its accuracy is promised for the given calculation range.

\section{Calculation}

Exemplary calculations have been made for $G=4500, \quad W=1.0 \mathrm{E}-03, \quad D=0.5 \mathrm{~nm} \quad$ and $\Delta_{n-2} / D=\Delta x / D=0.15$ respectively for the weak, medium and strong fluid-contact interactions.

The parameter $C_{y}$ is generally expressed as (Zhang 2014):

$C_{y}\left(H_{b f}\right)=a_{0}+\frac{a_{1}}{H_{b f}}+\frac{a_{2}}{H_{b f}^{2}}$

Where $a_{0}, a_{1}$ and $a_{2}$ are respectively constant, $H_{b f}=h_{b f} / h_{c r, b f}$, and $h_{c r, b f}$ is a critical thickness. Equation (5) is the equation fitting to the molecular dynamics simulation results which shows the correct variation of the effective viscosity of the adsorbed layer with the adsorbed layer thickness $h_{b f}$ if the values of $a_{0}, a_{1}$ and $a_{2}$ are properly chosen (Zhang 2014). For the weak fluid-contact interaction, the value of $C_{y}$ should be greater than but close to unity, and it should be slightly increased with the reduction of $h_{b f}$; For the medium fluid-contact interaction, the value of $C_{y}$ should be considerably greater than unity, and it should be more sensitively increased with the reduction of $h_{b f}$; For the strong fluid-contact interaction, the value of $C_{y}$ should be significantly greater than unity, and it should be the most sensitively increased with the reduction of $h_{b f}$. 
In the present calculation, for the weak, medium and strong fluid-contact interactions, the values of $a_{0}$, $a_{1}$ and $a_{2}$ are respectively shown in Table 1 . The other input parameter values are shown in Table 2.

Table 1 Fluid viscosity data for different fluid-contact interactions (Zhang 2014).

\begin{tabular}{|c|c|c|c|}
\hline $\begin{array}{c}\text { Parameter } \\
\text { Interaction }\end{array}$ & $a_{0}$ & $a_{1}$ & $a_{2}$ \\
\hline Strong & 1.8335 & -1.4252 & 0.5917 \\
\hline Medium & 1.0822 & -0.1758 & 0.0936 \\
\hline Weak & 0.9507 & 0.0492 & $\begin{array}{c}1.6447 \mathrm{E} \\
-04\end{array}$ \\
\hline
\end{tabular}

Table 2 Other input parameter values.

\begin{tabular}{|c|c|c|c|c|}
\hline $\begin{array}{c}\text { Parameter } \\
\text { Interaction }\end{array}$ & $m$ & $n$ & $q_{0}$ & $\begin{array}{c}h_{c r, b f} \\
(\mathrm{~nm})\end{array}$ \\
\hline Strong & 1.5 & 7 & 1.2 & 40 \\
\hline Medium & 1.0 & 5 & 1.1 & 20 \\
\hline Weak & 0.5 & 3 & 1.03 & 7 \\
\hline
\end{tabular}

For the present weak, medium and strong fluidcontact interactions, the thicknesses $h_{b f}$ of the adsorbed layer are respectively $1.65 \mathrm{~nm}, 2.76 \mathrm{~nm}$ and $4.32 \mathrm{~nm}$.

\section{Results}

Figure 2(a) shows the variations with the dimensionless rolling speed $U$ of the calculated values of $H_{c, t o t}$ for the medium fluid-contact interaction for different $k$ when $R=0.1 \mathrm{~mm}$. The results for the strong fluid-contact interaction are also compared with those for the conventional hydrodynamics. It is shown that for the medium fluid-contact interaction, the selection of the value of $k$ influences the calculated results of $H_{c, t o t}$ for low rolling speeds, however for sufficiently high rolling speeds such as greater than $1.0 \mathrm{E}-08$ this problem can be ignored. Higher the value of $k$, greater the calculated $H_{c \text {,tot }}$ value for low rolling speeds. It is reasonably shown that for the same operating condition, the value of $H_{c, t o t}$ for the medium fluidcontact interaction is greater than $H_{c, N}$ (for the conventional hydrodynamics without any adsorbed layer effect) but is lower than the value of $H_{c, t o t}$ for the strong fluid-contact interaction. It is estimated that for the medium fluid-contact interaction, the value of $k$ should be chosen as around 5.0. For sufficiently high rolling speeds, the adsorbed layer effect is shown to be negligible and the value of $H_{c, t o t}$ is close to $H_{c, N}$ for any type of the fluid contact interaction. However, for low rolling speeds, the adsorbed layer effect is shown to be not negligible and it increases the surface separation depending on the fluid-contact interaction. For a strong fluid-contact interaction, there seems to be a residual film in the contact at low rolling speeds due to the adsorbed layer effect; While for the conventional hydrodynamics, the surface separation $\left(H_{c, N}\right)$ drastically drops with the reduction of the low rolling speed.

Figure 2(b) shows the variations with the dimensionless rolling speed $U$ of the calculated values of $H_{c, t o t}$ for the weak fluid-contact interaction for different $k$ when $R=0.1 \mathrm{~mm}$. For better looking, the results for the strong fluid-contact interaction and for the conventional hydrodynamics are also shown. It is shown that for the weak fluidcontact interaction, the calculated value of $H_{c, t o t}$ depends on the value of $k$; For low rolling speeds, smaller the value of $k$, lower the value of $H_{c, t o t}$. However, for sufficiently high rolling speeds, it does not matter that which $k$ value is chosen for $1 \leq k \leq+\infty$, and the calculated $H_{c, t o t}$ value is close to $H_{c, N}$. It is estimated that for the weak fluidcontact interaction, the value of $k$ can also be chosen as around 5.0 when doing the present calculation. For the same operating condition, the calculated value of $H_{c, t o t}$ for the weak fluid-contact interaction is shown to be a little higher than $H_{c, N}$, but obviously lower than that of $H_{c, t o t}$ for the strong fluid-contact interaction for low rolling speeds.

Figure 3 shows the variations of the calculated values of $H_{c, t o t}$ with the dimensionless rolling speed $U$ respectively for the weak, medium and strong fluidcontact interactions when $R=0.01 \mathrm{~mm}$; For the weak and medium interactions, the calculation took $k=5.0$. The calculated values of $H_{c, t o t}$ are also compared with those of $H_{c, N}$. For $U<4.0 \mathrm{E}-09$, the values of $H_{c, t o t}$ were not plotted in Fig. 3 for any of these three fluid-contact interactions since the intermediate continuum fluid between the two adsorbed layers disappears in the Hertzian contact zone and there is only the very thin adsorbed layer remaining in this zone because of the low rolling speed and the present multiscale model fails. Figure 3 shows that for sufficiently low rolling speeds $(U)$ the fluid-contact interaction has a considerable influence on the surface separation for a given load, and stronger the fluid-contact interaction higher the value of $H_{c, t o t}$; However, for sufficiently high rolling speeds, the effect of the adsorbed layer is negligible whichever the fluid-contact interaction is, and the value of $H_{c, t o t}$ is nearly equal to $H_{c, N}$, which is calculated from the classical hydrodynamic theory. 


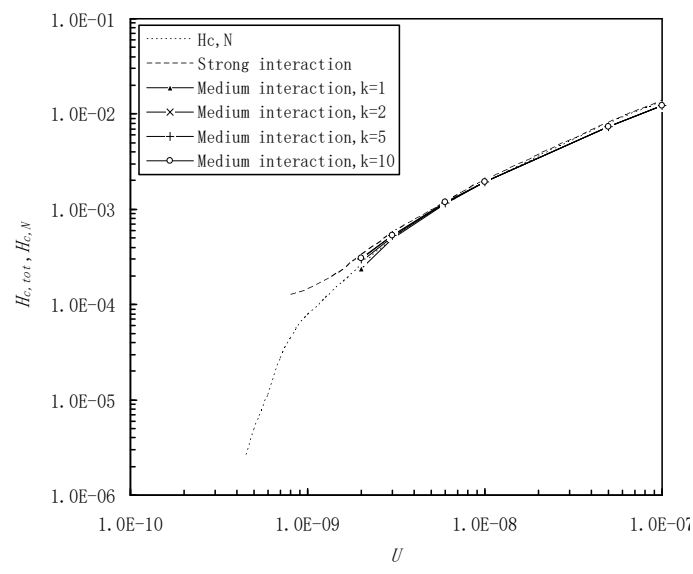

(a)

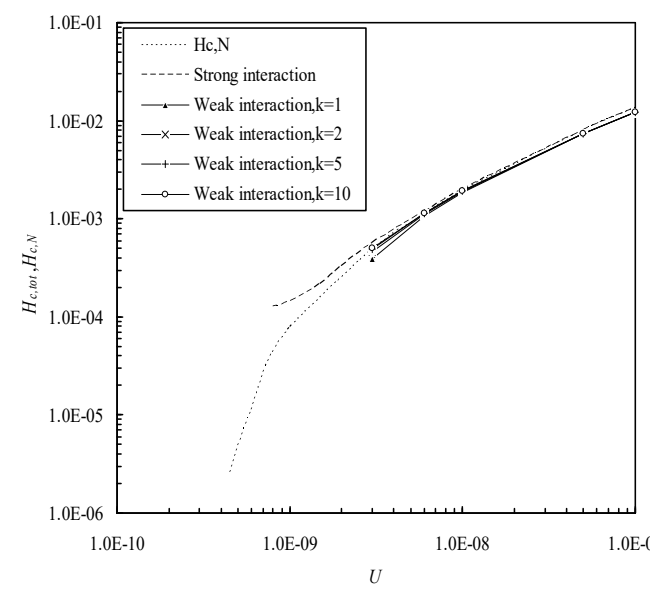

(b)

Fig. 2. Variations of the calculated values of $H_{c, t o t}$ and $H_{c, N}$ with the dimensionless rolling speed $U$ when $G=4500, W=1.0 \mathrm{E}-03$, and $R=0.1 \mathrm{~mm}$.

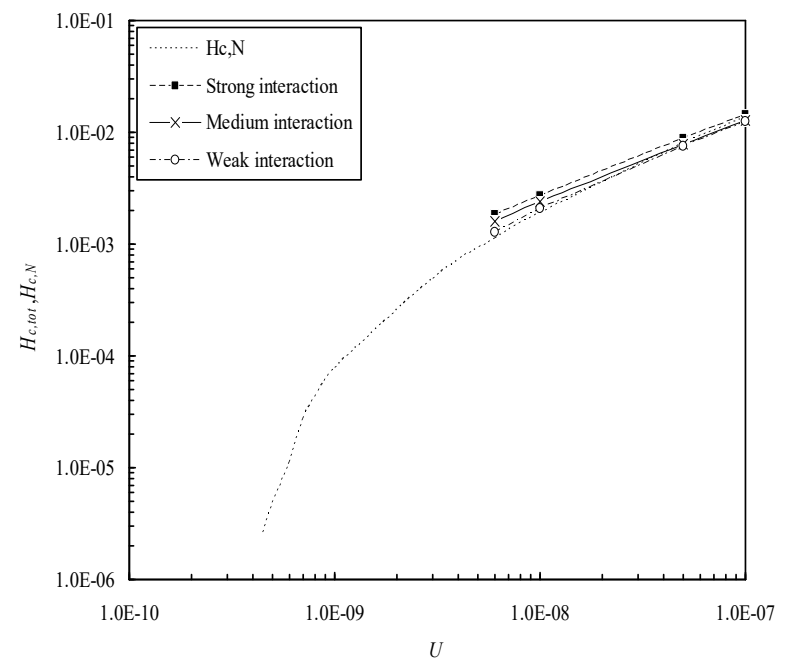

Fig. 3. Variations of the calculated values of $H_{c, t o t}$ and $H_{c, N}$ with the dimensionless rolling speed $U$ when $G=4500, W=1.0 \mathrm{E}-03$, and $R=0.01 \mathrm{~mm}$ (for the weak and medium interactions, $k=5.0$ ). Dashed line with data mark denotes $H_{c, t o t}$.

\section{CONCLUSIONS}

The multiscale calculations were made for the multiscale hydrodynamics in line contacts for different fluid-contact interactions based on the previously developed multiscale flow equations. It was found that for the medium and weak fluid-contact interactions, the present calculation should depend on the value of the parameter $k$, which is formulated in the important equation $\lambda_{b f, e}=h_{b f} /\left(k h_{c}\right)$; For a given load, smaller the value of $k$, lower the calculated value of the surface separation $H_{c, t o t}$. It is suggested that for these calculations the value of $k$ could be chosen as around 5.0.
The calculation results show that for the same operating condition, stronger the fluid-contact interaction, greater the surface separation for critically low rolling speeds; However, for sufficiently high rolling speeds, the adsorbed layer effect is negligible, and the hydrodynamics can be described by the conventional hydrodynamic theory. The obtained results are physically reasonable and important for the researches in this area. For a real case, if the input parameter values of the present model for the calculation as shown in Section 4 are correctly calibrated, the present model should be capable to give the correct results.

Although the present multiscale analysis and calculation are for the two-dimensional case, they are also valid for the three-dimensional case when the 
contact length is large enough so that the side leakage in the contact is negligible. This is true for the hydrodynamic lubricated line contacts in gears and roller bearings, both of which have the large axial contact lengths compared to the Hertzian contact widths. For other cases, even if the contact length is so small that the side leakage is not negligible, the present calculation can still be taken as the approximate solution.

The present multiscale hydrodynamic results are of significant interest to the design of mechanical engineering such as the design of rough surface lubrication on gears, bearings and cams etc. and the design of micro mechanical elements in micro machines

\section{REFERENCES}

Abraham, F. F. (1978). The interfacial density profile of a Lennard-Jones fluid in contact with a (100) Lennard-Jones wall and its relationship to idealized fluid/wall systems: A Monte Carlo simulation. Journal of Chemical Physics 68, 3713-3716.

Begelinger, A. and A. W. J. de Gee (1976). On the mechanism of lubricant film failure in sliding concentrated steel contacts. Journal of Tribology 98(4), 575.

Bitsanis, I., J. J. Magda, M. Tirrell and H. T. Davis (1987). Molecular dynamics of flow in micropores. Journal of Chemical Physics 87(3), 1733-1750.

Bocquet, L. and E. Charlaix (2010). Nanoflfluidics, from bulk to interfaces. Chemical Society Reviews 39, 1073-1095.

Calabrò, F., K. P. Lee and D. Mattia (2013). Modelling flow enhancement in nanochannels: viscosity and slippage. Applied Mathematics Letters 26(10), 991-994.

Chauveteau, G., M. Tirrell and A. Omari (1984). Concentration dependence of the effective viscosity of polymer solutions in small pores with repulsive or attractive walls. Journal of Colloid and Interface Science 100, 41-54.

Horn, R. G., D. T. Smith and W. Haller (1989). Surface forces and viscosity of water measured between silica sheets. Chemical Physics Letters 162, 404-408.

Jabbarzadeh, A., J. D. Atkinson and R. I. Tanner (1997). Rheological properties of thin liquid films by molecular dynamics simulations. Journal of Non-Newtonian Fluid Mechanics 69(2-3), 169-193.

Mattia, D. and F. Calabro(2012). Explaining high flow rate of water in carbon nanotubes via solidliquid molecular interactions. Microfluidics and Nanofluidics 13(1), 125-130.
Meyer, E., R. M. Overney, K. Dransfeld and T. Gyalog (1998). Nanoscience-Friction and Rheology on the Nanometer Scale. World Scientific, New Jersey.

Ritos, K., D. Mattia, F. Calabro and J. M. Reese (2014). Flow enhancement in nanotubes of different materials and lengths. Journal of Chemical Physics 40, 014702.

Sbragaglia, M., R. Benzi, L. Biferale, S. Succi and F. Toschi (2006). Surface roughnesshydrophobicity coupling in microchannel and nano- channel flows. Physical Review Letters 97, 204503.

Sofos, F., T. E. Karakasidis and A. Liakopoulos (2009). Transport properties of liquid argon in krypton nanochannels: Anisotropy and nonhomogeneity introduced by the solid walls. International Journal of Heat and Mass Transfer 52, 735-743.

Sofos, D. F., T. E. Karakasidis and A. Liakopoulos (2010). Effect of wall roughness on shear viscosity and diffusion in nanochannels. International Journal of Heat and Mass Transfer 53, 3839-3846.

Sofos, F., T. E. Karakasidis and A. Liakopoulos (2013). Parameters affecting slip length at the nanoscale. Journal of Computational and Theoretical Nanoence 10(3), 648-650.

Zhang, Y. B. (2005). Review of mixed lubrication in concentrated contacts: Thinning, mixed and partial films, classical, modern and future modes. International Journal for Engineering Modeling 18, 87-129.

Zhang, Y. B. (2006). Flow factor of non-continuum fluids in one-dimensional contact. Industrial Lubrication and Tribology 58(3), 151-169.

Zhang, Y. B. (2013). New film thickness equation for elastohydrodynamic lubrication of isothermal smooth line contacts under heavy loads for newtonian fluids. Advanced Science, Engineering and Medicine 5(2), 182-186.

Zhang, Y. B. (2014). Lubrication analysis for a line contact covering from boundary lubrication tohydrodynamic lubrication: Part I- Micro contact results. Journal of Computational and Theoretical Nanoscience 11(1), 62-70.

Zhang, Y. B. (2016). The flow equation for a nanoscale fluid flow. International Journal of Heat and Mass Transfer 92, 1004-1008.

Zhang, Y. B. (2020). Modeling of flow in a very small surface separation. Applied Mathematical Modelling 82, 573-586.

Zhang, Y. B. (2021). Multiscale hydrodynamics in line contacts. Mechanics Research Communications 111, 103658. 